Supplement of Atmos. Chem. Phys., 21, 12989-13010, 2021

https://doi.org/10.5194/acp-21-12989-2021-supplement

(C) Author(s) 2021. CC BY 4.0 License.

(c) (i)

Supplement of

\title{
Optical properties of coated black carbon aggregates: numerical simulations, radiative forcing estimates, and size-resolved parameterization scheme
}

Baseerat Romshoo et al.

Correspondence to: Baseerat Romshoo (baseerat@tropos.de)

The copyright of individual parts of the supplement might differ from the article licence. 

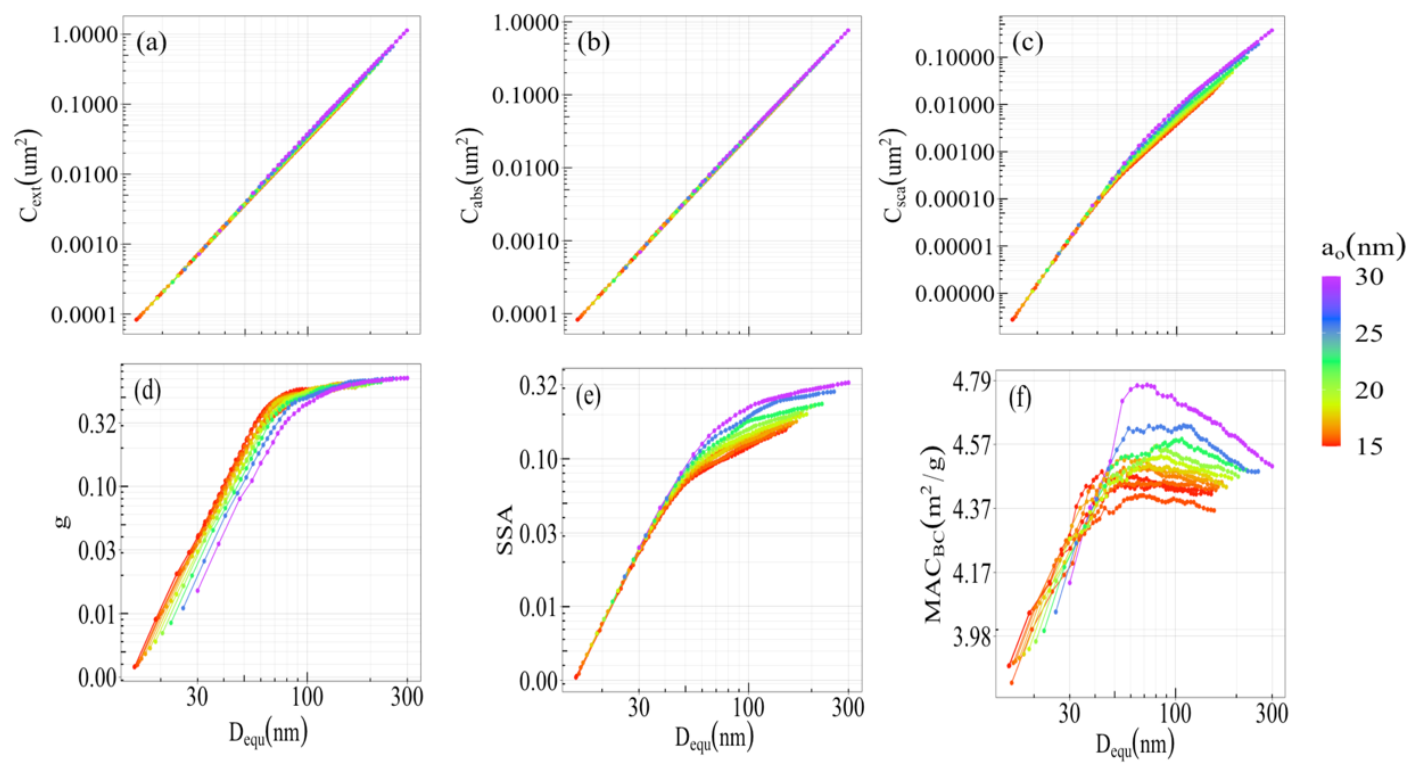

Figure S1. Optical properties of pure BCFAs for various radii of primary particles $\left(a_{0}\right)$ as a function of the volume equivalent radius ( $R_{\text {equ }}$ ): (a) extinction cross-section $C_{\text {ext }}$, (b) absorption cross-section $C_{\text {abs, }}$, (c) scattering cross-
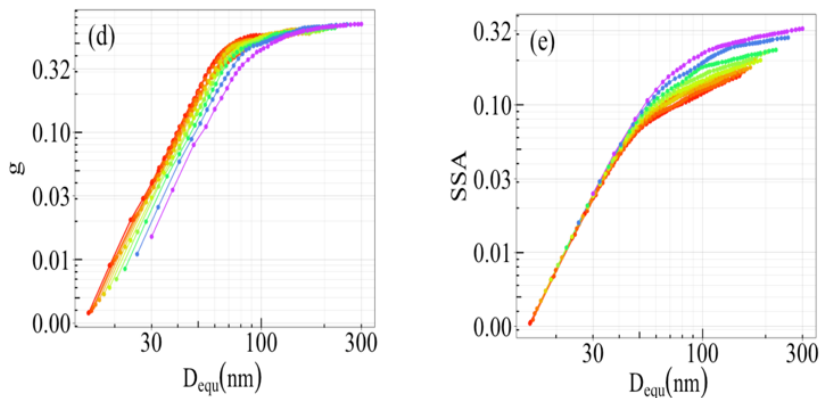
section $C_{\text {sca }}$, (d) asymmetry parameter $g$, (e) single scattering albedo $S S A$, and (f) black carbon mass absorption cross-section $M A C_{\mathrm{BC}}$ at $\lambda=660 \mathrm{~nm}$.
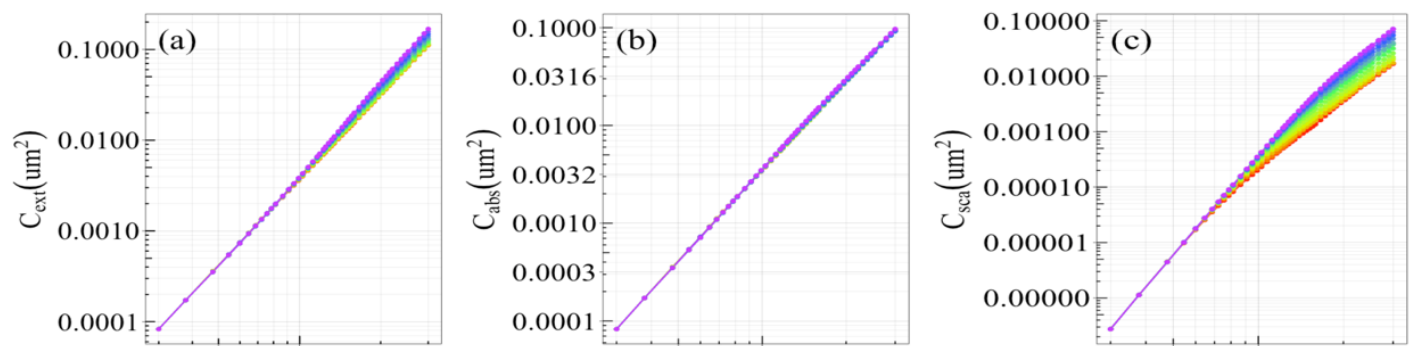

$\mathrm{D}_{\mathrm{f}}$
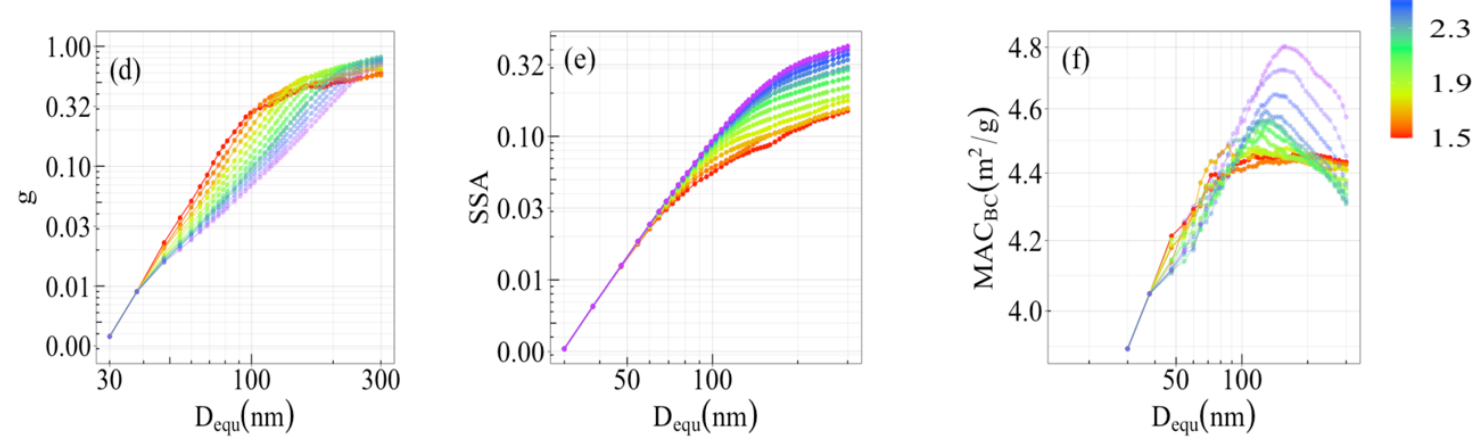

Figure S2. Optical properties of pure BCFAs as a function of volume equivalent radius $\left(R_{\text {equ }}\right)$ for various fractal dimension $\left(D_{\mathrm{f}}\right)$ : (a) extinction cross-section $C_{\text {ext }}$, (b) absorption cross-section $C_{\text {abs, }}$, (c) scattering cross-section $C_{\text {sca, }}$ (d) asymmetry parameter $g$, (e) single scattering albedo $S S A$, and (f) black carbon mass absorption cross-section $M A C_{\mathrm{BC}}$ at $\lambda=660 \mathrm{~nm}$. 

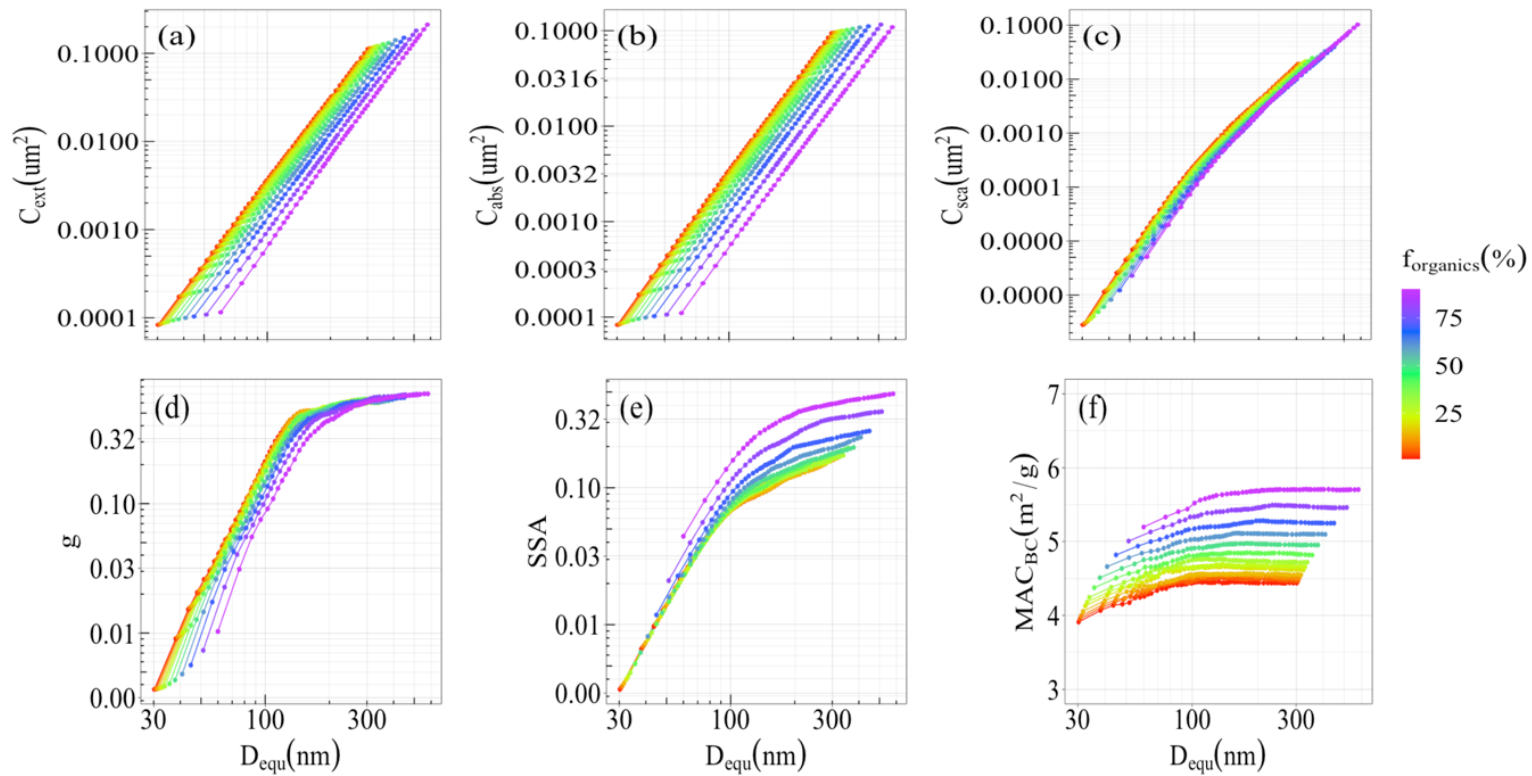

15 Figure S3. Optical properties of BCFAs $\left(D_{\mathrm{f}}=1.7\right)$ as a function of volume equivalent radius $\left(R_{\text {equ }}\right)$ for various fraction of organics $\left(f_{\text {organics }}\right)$ : (a) extinction cross-section $C_{\text {ext }}$, (b) absorption cross-section $C_{\text {abs, }}$ (c) scattering cross-section $C_{\text {sca }}$ (d) asymmetry parameter $g$, (e) single scattering albedo $S S A$, and (f) black carbon mass absorption cross-section $M A C_{\mathrm{BC}}$ at $\lambda=660 \mathrm{~nm}$.
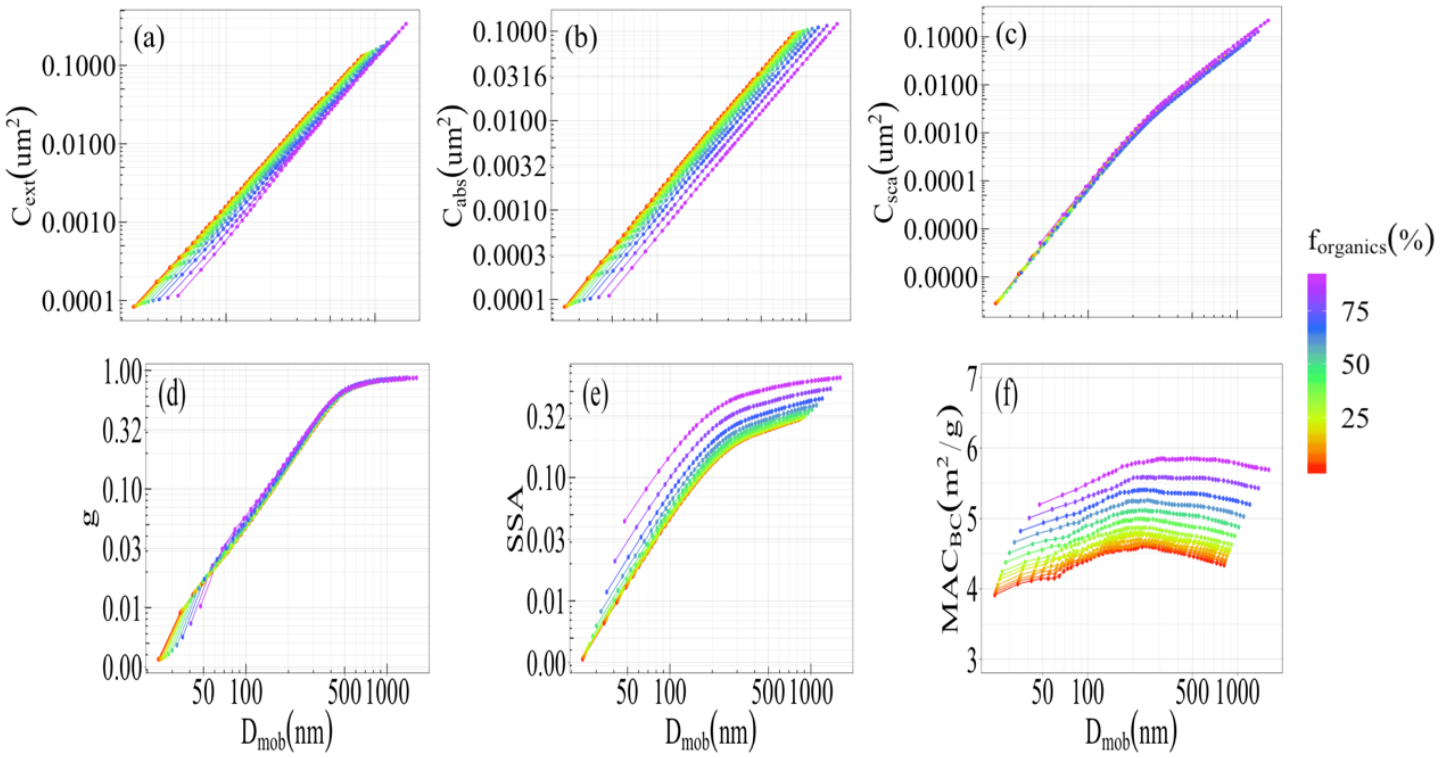

Figure S4. Optical properties of BCFAs $\left(D_{\mathrm{f}}=2.2\right)$ as a function of $D_{\text {mob }}$ for various fraction of organics $\left(f_{\text {organics }}\right)$ : (a) extinction cross-section $C_{\text {ext }}$, (b) absorption cross-section $C_{\text {abs, }}$, (c) scattering cross-section $C_{\text {sca, }}$, (d) asymmetry parameter $g$, (e) single scattering albedo $S S A$, and (f) black carbon mass absorption cross-section $M A C_{\mathrm{BC}}$ at $\lambda=$ $660 \mathrm{~nm}$. 

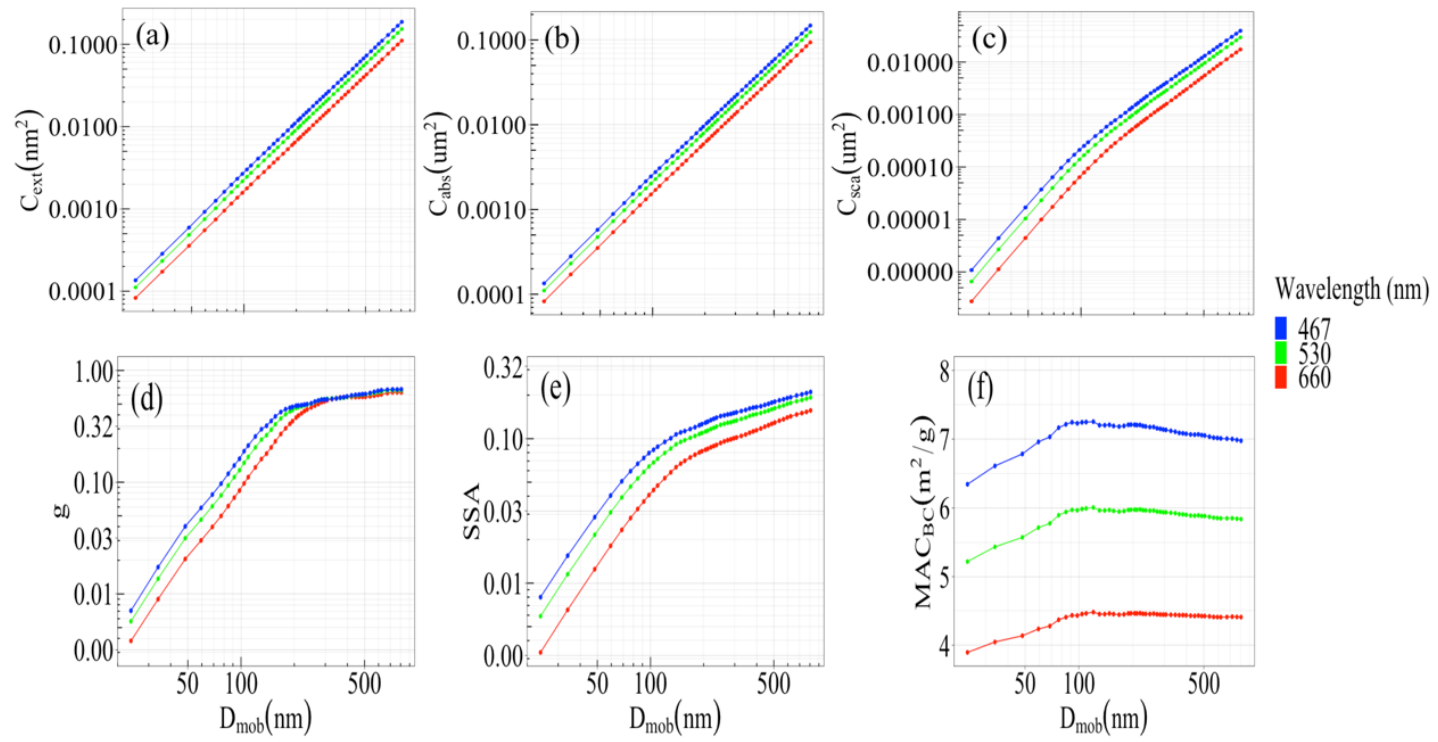

Figure S5. Optical properties of pure BCFAs $\left(D_{\mathrm{f}}=1.7\right)$ as a function of $D_{\text {mob }}$ for various wavelengths $(\lambda)$ : (a) extinction cross-section $C_{\text {ext }}$, (b) absorption cross-section $C_{\text {abs, }}$, (c) scattering cross-section $C_{\text {sca, }}$ (d) asymmetry parameter $g$, (e) single scattering albedo $S S A$, and (f) black carbon mass absorption cross-section $M A C_{\mathrm{BC}}$.

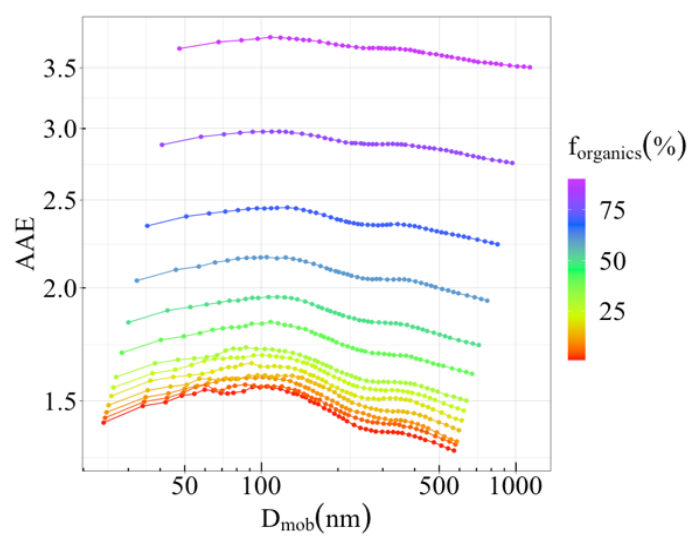

Figure S6. Ångström Absorption Exponent (AAE) of slightly compact BCFAs $\left(D_{\mathrm{f}}=2.2\right)$ with changing fraction of organics $\left(f_{\text {organics }}\right)$ and mobility diameter $\left(D_{\text {mob }}\right)$.

\section{Bulk optical properties of BCFAs}

40 For application over the atmosphere, it is more meaningful to know the averaged optical properties over a certain particle size distribution, i.e. the bulk optical properties. Bulk optical properties of BC are useful since they can be used directly for simulations of radiative forcing. The bulk optical properties of BCFAs are calculated with an assumption that the mobility diameter $D_{m o b}$ of BCFAs follow a lognormal size distribution:

$n\left(D_{m o b}\right)=\frac{1}{\sqrt{\pi D_{m o b}} \ln (\sigma)} \exp \left[-\left[\frac{\ln \left(D_{m o b} / 2\right)-\ln \left(D_{m o b} / 2\right)}{\sqrt{2} \ln (\sigma)}\right]^{2}\right]$,

(1)

where $D_{m o b_{o}}$ is the geometric mean mobility diameter, and $\sigma$ is the standard deviation in $\ln \left(D_{m o b}\right)$.

Table 4 shows the bulk optical properties of BCFAs for various compositions and morphologies at a wavelength of $530 \mathrm{~nm}$. For each case, the bulk optical properties are calculated as integrals over the lognormal size distribution $n\left(D_{m o b}\right)$ with limits of $D_{m o b}$ varying from 0.030 to $0.60 \mu \mathrm{m}$, and the corresponding MSTM calculated values. The calculations are done following the mathematical formulas summarised in Li et al (2016). 
Table S1. Bulk optical properties of black carbon for different fraction of organics $\left(f_{\text {organics }}\right)$ and fractal dimension

\begin{tabular}{|c|c|c|c|c|c|c|c|c|c|c|c|c|c|c|}
\hline & \multicolumn{14}{|c|}{$f_{\text {organics }}(\%)$} \\
\hline & 0 & 1 & 5 & 10 & 15 & 20 & 25 & 30 & 40 & 50 & 60 & 70 & 80 & 90 \\
\hline \multicolumn{15}{|l|}{$D_{\mathrm{f}}=1.7$} \\
\hline$C_{\text {ext }}\left(\times 10^{-2} \mu \mathrm{m}^{-2}\right)$ & 2.94 & 2.96 & 3.02 & 3.03 & 3.04 & 3.12 & 3.12 & 3.14 & 3.18 & 3.14 & 3.08 & 2.92 & 2.74 & 2.35 \\
\hline$C_{\text {abs }}\left(\times 10^{-2} \mu \mathrm{m}^{-2}\right)$ & 2.54 & 2.55 & 2.58 & 2.60 & 2.62 & 2.65 & 2.66 & 2.67 & 2.66 & 2.60 & 2.48 & 2.25 & 1.86 & 1.35 \\
\hline$C_{\text {sca }}\left(\times 10^{-2} \mu \mathrm{m}^{-2}\right)$ & 0.40 & 0.41 & 0.44 & 0.43 & 0.42 & 0.47 & 0.47 & 0.47 & 0.52 & 0.54 & 0.61 & 0.67 & 0.88 & 1.00 \\
\hline$S S A$ & 0.14 & 0.14 & 0.15 & 0.14 & 0.14 & 0.15 & 0.15 & 0.15 & 0.16 & 0.17 & 0.20 & 0.23 & 0.32 & 0.43 \\
\hline$g$ & 0.60 & 0.58 & 0.57 & 0.60 & 0.59 & 0.59 & 0.61 & 0.57 & 0.62 & 0.60 & 0.60 & 0.62 & 0.64 & 0.63 \\
\hline \multicolumn{15}{|l|}{$D_{\mathrm{f}}=2.2$} \\
\hline$C_{\text {ext }}\left(\times 10^{-2} \mu \mathrm{m}^{-2}\right)$ & 0.03 & 0.03 & 0.03 & 0.04 & 0.04 & 0.04 & 0.04 & 0.04 & 0.04 & 0.04 & 0.04 & 0.04 & 0.03 & 0.03 \\
\hline$C_{\mathrm{abs}}\left(\times 10^{-2} \mu \mathrm{m}^{-2}\right)$ & 0.03 & 0.03 & 0.03 & 0.03 & 0.03 & 0.03 & 0.03 & 0.03 & 0.03 & 0.03 & 0.02 & 0.02 & 0.02 & 0.01 \\
\hline$C_{\text {sca }}\left(\times 10^{-2} \mu \mathrm{m}^{-2}\right)$ & 0.01 & 0.01 & 0.01 & 0.01 & 0.01 & 0.01 & 0.01 & 0.01 & 0.01 & 0.01 & 0.01 & 0.01 & 0.02 & 0.02 \\
\hline$S S A$ & 0.25 & 0.26 & 0.26 & 0.26 & 0.26 & 0.27 & 0.27 & 0.27 & 0.29 & 0.31 & 0.33 & 0.37 & 0.45 & 0.57 \\
\hline$G$ & 0.70 & 0.69 & 0.70 & 0.70 & 0.71 & 0.71 & 0.72 & 0.72 & 0.73 & 0.73 & 0.75 & 0.76 & 0.77 & 0.75 \\
\hline
\end{tabular}

\section{Parametrization for bulk optical properties}

60 An important application of the optical parametrization scheme would be calculation of the bulk optical properties of atmospheric BC aerosols. The bulk optical properties can be calculated between any limits of mobility diameter $\left(D_{\text {mob }}\right)$, for the cases of fractal dimension $\left(D_{\mathrm{f}}\right)$, fraction of organics $\left(f_{\text {organics }}\right)$, and wavelength $(\lambda)$ provided in the parametrization scheme. Following Eq. (1), it must be assumed that the mobility diameter $\left(D_{\mathrm{mob}}\right)$ of the BCFAs follow a log normal distribution:

65

$n\left(D_{m o b}\right)=\frac{1}{\sqrt{2 \pi D_{m o b}} \ln (\sigma)} \exp \left[-\left[\frac{\ln \left(D_{m o b}\right)-\ln \left(\overline{D_{m o b}}\right)}{\sqrt{2} \ln (\sigma)}\right]^{2}\right]$

where $\overline{D_{m o b}}$ is the arithmetic mean diameter, and $\sigma$ is the standard deviation in $\ln \left(D_{m o b}\right)$.

70 The bulk extinction cross-section is given by:

$\left\langle C_{\text {ext }}\right\rangle=\int_{D_{m o b}^{\min }}^{D_{\text {max }}^{\max }} C_{\text {ext }}\left(D_{\text {mob }}, D_{f}, f_{\text {organics }}, \lambda\right) n\left(D_{\text {mob }}\right) d\left(D_{\text {mob }}\right)$,

using the parametrization scheme for extinction cross-section,

75

$\left\langle C_{e x t}\right\rangle=\int_{D_{m o b}^{\operatorname{mob}}}^{D_{m a x}^{\max }}\left(e^{c_{0}}+e^{c_{1}} D_{m o b}\right) n\left(D_{m o b}\right) d\left(D_{m o b}\right)$.

The values of the coefficients $C_{0}$ and $C_{1}$ must be chosen from their tabulated values for various cases of fractal dimension $\left(D_{\mathrm{f}}\right)$, fraction of organics $\left(f_{\text {organics }}\right)$, and wavelength $(\lambda)$ provided in the section of supplementary below.

80 Similarly, using the parametrization schemes provided, the corresponding bulk optical properties can be calculated as:

$\left\langle C_{a b s}\right\rangle=\int_{D_{m o b}^{\min }}^{D_{m a x}^{\max }}\left(e^{g_{0}}+e^{g_{1}} D_{m o b}\right) n\left(D_{m o b}\right) d\left(D_{m o b}\right)$,

$85\left\langle C_{s c a}\right\rangle=\int_{D_{m o b}^{m o b}}^{D_{m a x}^{\max }}\left(e^{H_{0}}+e^{H_{1}} D_{m o b}+e^{H_{2}} \ln D_{m o b}\right) n\left(D_{m o b}\right) d\left(D_{m o b}\right)$,

$\langle S S A\rangle=\int_{D_{m o b}^{m i n}}^{D_{m a x}^{\max }}\left(e^{k_{0}}+e^{k_{1}} D_{m o b}+e^{k_{2}} \ln D_{m o b}\right) n\left(D_{m o b}\right) d\left(D_{m o b}\right)$

$\langle g\rangle=\int_{D_{m o b}^{\min }}^{D_{m a x}^{\max }}\left(e^{s_{0}}+e^{s_{1}} D_{m o b}+e^{s_{2}} D_{m o b}^{2}+e^{s_{3}} D_{m o b}^{3}\right) n\left(D_{m o b}\right) d\left(D_{m o b}\right)$. 
The relative errors between the bulk optical properties from the MSTM calculations and the parametrization scheme are comparable to that in the case of single sized BCFAs shown in the section 3.7.1. 


\section{Parametrization scheme for optical properties of BCFAs}

$\ln C_{\text {ext }}=c_{0}+c_{1} \ln D_{m o b}$

$\ln C_{a b s}=g_{0}+g_{1} \ln D_{m o b}$

$\ln C_{s c a}=H_{0}+H_{1} \ln D_{m o b}+H_{2} \ln \left(\ln D_{m o b}\right)$

$\ln S S A=k_{0}+k_{1} \ln D_{m o b}+k_{2} \ln \left(\ln D_{m o b}\right)$

110

$\operatorname{lng}=\sum_{n=0}^{3} s_{n} \ln D_{m o b}^{n}$

Table S2. Parameterization scheme for coated and non-coated black carbon fractal aggregates (BCFA).

\begin{tabular}{|c|c|c|c|c|c|c|c|c|c|c|c|c|c|c|c|c|}
\hline$\lambda$ & $\mathbf{D}_{\mathrm{f}}$ & $f_{\text {organics }}$ & c0 & c1 & g0 & g1 & H0 & H1 & H2 & s0 & s1 & s2 & s3 & k0 & k1 & k2 \\
\hline 660 & 1.5 & 0 & -15.7525 & 2.0244 & -15.4847 & 1.9605 & -24.2159 & 1.9921 & 3.5801 & -46.0080 & 21.3332 & -3.3873 & 0.1816 & -8.8739 & -0.1242 & 4.1179 \\
\hline 660 & 1.5 & 5 & -15.7550 & 2.0214 & -15.5059 & 1.9597 & -31.3601 & 0.2872 & 13.3115 & 0.6842 & -2.4337 & 0.6085 & -0.0406 & -14.9613 & -1.5899 & 12.4673 \\
\hline 660 & 1.5 & 25 & -15.8438 & 2.0177 & -15.6260 & 1.9616 & -24.6220 & 1.6677 & 4.8432 & 9.8861 & -7.6666 & 1.5889 & -0.1010 & -8.5946 & -0.3101 & 4.6065 \\
\hline 660 & 1.5 & 50 & -16.1005 & 2.0298 & -15.8183 & 1.9591 & -21.4000 & 2.3674 & 0.6833 & -33.6550 & 14.4609 & -2.1386 & 0.1072 & -6.1086 & 0.1704 & 1.6966 \\
\hline 660 & 1.5 & 70 & -16.4923 & 2.0594 & -16.1146 & 1.9624 & -26.7081 & 1.2486 & 7.4745 & -22.5955 & 8.5255 & -1.0988 & 0.0475 & -10.3414 & -0.8352 & 7.6265 \\
\hline 660 & 1.6 & 0 & -15.7675 & 2.0278 & -15.4998 & 1.9626 & -30.8563 & 0.4502 & 12.5063 & -51.4395 & 23.8510 & -3.7532 & 0.1980 & -14.7285 & -1.4969 & 12.0341 \\
\hline 660 & 1.6 & 5 & -15.7611 & 2.0214 & -15.5484 & 1.9657 & -26.0321 & 1.3267 & 6.8446 & -67.6214 & 31.3213 & -4.9038 & 0.2572 & -10.3641 & -0.7155 & 6.9666 \\
\hline 660 & 1.6 & 25 & -15.8199 & 2.0167 & -15.5835 & 1.9543 & -29.1829 & 0.5997 & 11.0671 & 15.0126 & -11.1566 & 2.3105 & -0.1478 & -13.2172 & -1.3853 & 10.8791 \\
\hline 660 & 1.6 & 50 & -16.0327 & 2.0207 & -15.7920 & 1.9548 & -23.5291 & 1.7441 & 4.0211 & -28.7900 & 10.8963 & -1.3692 & 0.0558 & -7.9102 & -0.3621 & 4.5393 \\
\hline 660 & 1.6 & 70 & -16.3849 & 2.0426 & -16.0953 & 1.9595 & -23.9888 & 1.6021 & 4.7863 & -52.7974 & 22.7540 & -3.3130 & 0.1616 & -7.2572 & -0.3732 & 4.3668 \\
\hline 660 & 1.7 & 0 & -15.6665 & 2.0113 & -15.4404 & 1.9526 & -22.7664 & 2.0195 & 2.7341 & -135.7843 & 65.3114 & -10.5239 & 0.5659 & -7.4564 & -0.0716 & 3.2013 \\
\hline 660 & 1.7 & 1 & -15.7172 & 2.0183 & -15.4884 & 1.9588 & -25.8963 & 1.3457 & 6.7473 & -122.5711 & 58.9261 & -9.5008 & 0.5114 & -10.3542 & -0.7121 & 6.9776 \\
\hline 660 & 1.7 & 5 & -15.7239 & 2.0186 & -15.4929 & 1.9569 & -31.7647 & -0.0265 & 14.6745 & -50.8588 & 22.2507 & -3.3079 & 0.1656 & -15.2113 & -1.8591 & 13.5868 \\
\hline 660 & 1.7 & 10 & -15.7602 & 2.0205 & -15.5022 & 1.9557 & -23.8127 & 1.8851 & 3.7649 & -159.7634 & 77.9977 & -12.7479 & 0.6950 & -8.0993 & -0.1458 & 3.8261 \\
\hline 660 & 1.7 & 15 & -15.7845 & 2.0191 & -15.5576 & 1.9598 & -23.2942 & 1.9081 & 3.3738 & -133.6831 & 64.5100 & -10.4305 & 0.5627 & -7.8428 & -0.1847 & 3.8075 \\
\hline 660 & 1.7 & 20 & -15.7825 & 2.0157 & -15.5614 & 1.9552 & -32.8077 & -0.2625 & 16.0185 & -36.1842 & 14.4067 & -1.9131 & 0.0833 & -16.1224 & -2.0803 & 14.8498 \\
\hline 660 & 1.7 & 25 & -15.8531 & 2.0220 & -15.6139 & 1.9591 & -28.1449 & 0.8408 & 9.6764 & -100.6791 & 47.1922 & -7.4289 & 0.3908 & -11.9529 & -1.1075 & 9.2395 \\
\hline 660 & 1.7 & 30 & -15.8519 & 2.0163 & -15.6197 & 1.9546 & -23.9360 & 1.7146 & 4.3721 & -129.5501 & 61.9130 & -9.9117 & 0.5292 & -8.5159 & -0.3948 & 4.9260 \\
\hline 660 & 1.7 & 40 & -16.0243 & 2.0333 & -15.7297 & 1.9594 & -25.4089 & 1.5384 & 5.7834 & -86.7096 & 40.5283 & 706 & 0.3349 & -9.4150 & 013 & 5.8219 \\
\hline 660 & 1.7 & 50 & -16.0639 & 2.0259 & -15.8239 & 1.9597 & -25.9052 & 1.2578 & 6.9899 & -80.3566 & 36.9175 & -5.7005 & 0.2940 & -9.5129 & -0.7003 & 6.5787 \\
\hline 660 & 1.7 & 60 & -16.2345 & 2.0381 & -15.9518 & 1.9608 & -26.0324 & 1.2540 & 7.0923 & -52.0232 & 23.3486 & -3.5458 & 0.1804 & -9.6134 & -0.7470 & 6.8648 \\
\hline 660 & 1.7 & 70 & -16.3536 & 2.0390 & -16.1041 & 1.9606 & -32.1653 & -0.0616 & 14.9674 & -21.9360 & 8.4632 & -1.1023 & 0.0475 & -13.6578 & -1.6823 & 12.3611 \\
\hline 660 & 1.7 & 80 & -16.7395 & 2.0821 & -16.3304 & 1.9612 & -38.0200 & -0.9987 & 21.4747 & 3.1163 & -3.3064 & 0.7257 & -0.0464 & -16.6107 & -2.2156 & 15.9668 \\
\hline 660 & 1.7 & 90 & -16.9556 & 2.1004 & -16.6020 & 1.9615 & -23.2061 & 1.5596 & 4.7601 & -47.3551 & 19.7729 & -2.7880 & 0.1317 & -5.4062 & -0.3913 & 3.7885 \\
\hline 660 & 1.8 & 0 & -15.7101 & 2.0220 & -15.4458 & 1.9541 & -23.8616 & 1.7819 & 4.2225 & -137.0045 & 64.2293 & -10.0778 & 0.5276 & -8.1299 & -0.2353 & 4.1941 \\
\hline
\end{tabular}




\begin{tabular}{|c|c|c|c|c|c|c|c|c|c|c|c|c|c|c|c|c|}
\hline$\lambda$ & $\mathbf{D}_{\mathrm{f}}$ & $f_{\text {organics }}$ & c0 & c1 & g0 & g1 & H0 & H1 & H2 & s0 & s1 & s2 & s3 & k0 & k1 & k2 \\
\hline 660 & 1.8 & 5 & -15.7925 & 2.0314 & -15.4917 & 1.9562 & -27.5406 & 1.0574 & 8.7186 & -115.6974 & 53.8541 & -8.4068 & 0.4385 & -11.5420 & -0.9278 & 8.4485 \\
\hline 660 & 1.8 & 25 & -15.8439 & 2.0232 & -15.5996 & 1.9570 & -32.3807 & -0.1483 & 15.4265 & -119.8288 & 56.5257 & -8.9570 & 0.4748 & -15.0559 & -1.8492 & 13.5171 \\
\hline 660 & 1.8 & 50 & -16.0872 & 2.0337 & -15.7961 & 1.9554 & -28.3066 & 0.7812 & 10.0090 & -126.5501 & 58.8226 & -9.1555 & 0.4755 & -11.5542 & -1.1150 & 9.1759 \\
\hline 660 & 1.8 & 70 & -16.4100 & 2.0521 & -16.0932 & 1.9587 & -30.4162 & 0.3195 & 12.7735 & -55.0677 & 23.9062 & -3.4960 & 0.1710 & -12.5547 & -1.4507 & 11.0171 \\
\hline 660 & 1.9 & 0 & -15.6963 & 2.0220 & -15.4085 & 1.9471 & -30.0008 & 0.3678 & 12.4687 & -101.7978 & 45.3518 & -6.7572 & 0.3355 & -13.6819 & -1.5148 & 11.6529 \\
\hline 660 & 1.9 & 5 & -15.7176 & 2.0224 & -15.4146 & 1.9440 & -33.1895 & -0.3221 & 16.5742 & -81.5721 & 35.1277 & -5.0436 & 0.2400 & -16.9354 & -2.2243 & 15.8707 \\
\hline 660 & 1.9 & 25 & -15.8518 & 2.0280 & -15.5323 & 1.9458 & -26.7767 & 1.1206 & 8.1099 & -90.0003 & 39.0629 & -5.6576 & 0.2724 & -10.8130 & -0.8831 & 7.9656 \\
\hline 660 & 1.9 & 50 & -16.0837 & 2.0376 & -15.7752 & 1.9522 & -32.2508 & -0.0935 & 15.2303 & -108.9910 & 48.9904 & -7.3835 & 0.3718 & -14.2591 & -1.7368 & 12.8407 \\
\hline 660 & 1.9 & 70 & -16.4636 & 2.0654 & -16.0805 & 1.9572 & -29.8972 & 0.4238 & 12.1912 & -154.7034 & 71.3913 & -11.0253 & 0.5684 & -11.4088 & -1.2381 & 9.7067 \\
\hline 660 & 2 & 0 & -15.7288 & 2.0320 & -15.3580 & 1.9389 & -34.4017 & -0.5381 & 18.0565 & -33.7398 & 9.7380 & -0.6146 & -0.0142 & -17.6773 & -2.3471 & 16.7518 \\
\hline 660 & 2 & 5 & -15.8193 & 2.0434 & -15.4130 & 1.9436 & -34.9233 & -0.5664 & 18.4429 & -35.2785 & 10.9254 & -0.8826 & 0.0045 & -17.9426 & -2.3493 & 16.9198 \\
\hline 660 & 2 & 25 & -15.9172 & 2.0421 & -15.5 & 1.9454 & -31.4270 & 0.1639 & 13.9926 & -63 & 24.9933 & -3.1927 & 0.1307 & -14.8040 & -1.7246 & 825 \\
\hline 660 & 2 & 50 & & 2.0577 & -15.7 & 1.9513 & & & 11.0481 & & & & & -11.9699 & -1.1972 & 992 \\
\hline 660 & 2 & 70 & -16 & 2.0789 & -16.0 & 1.9505 & -30 & 0.4 & 12.5 & & & -7. & & -11.7531 & -1.2834 & 10.1079 \\
\hline 660 & 2.1 & 0 & 414 & 2.0562 & -15.3 & 1.9359 & & -1.5 & 24.4085 & 17 & -15 & & & 66 & -3.0761 & 035 \\
\hline 660 & 2.1 & 5 & -15.8896 & 2.0600 & -15.3 & 1.9383 & -39 & -1.5065 & 24.3083 & & -13.1479 & & & 129 & -3.1112 & 459 \\
\hline 660 & 2.1 & 25 & -15.9769 & 2.0580 & -15.4672 & 1.9354 & & -0.8448 & 20.3844 & & & 768 & 200 & 2591 & -2.5949 & 18.5602 \\
\hline 660 & 2.1 & 50 & -16.2544 & 2.0752 & -15.7219 & 1.9439 & -31.6634 & 0.2219 & 13.9815 & -64.7328 & 25.0202 & -3.1550 & 0.1280 & -14.3508 & -1.6346 & 563 \\
\hline 660 & 2.1 & 70 & -16.6360 & 2.1070 & -16.0171 & 1.9477 & -31.2855 & 0.2983 & 13.5405 & -106.9849 & 45.9556 & -6.6040 & 0.3168 & -12.6759 & -1.4254 & 11.1524 \\
\hline 660 & 2.2 & 0 & -15.9386 & 2.0773 & -15.2911 & 1.9283 & -42.5466 & -2.0281 & 27.7710 & 45.7660 & -30.3926 & 6.0367 & -0.3769 & -24.0551 & -3.5336 & 24.4260 \\
\hline 660 & 2.2 & 1 & -15.9574 & 2.0798 & -15.3008 & 1.9291 & -43.5919 & -2.2444 & 29.0821 & 46.5315 & -30.6360 & 6.0537 & -0.3766 & -24.6930 & -3.6613 & 25.2133 \\
\hline 660 & 2.2 & 5 & -15.9792 & 2.0807 & -15.3260 & 1.9305 & -43.1870 & -2.1503 & 28.5326 & 43.4108 & -29.0778 & 5.7948 & -0.3623 & -24.2837 & -3.5754 & 24.6981 \\
\hline 660 & 2.2 & 10 & -16.0323 & 2.0866 & -15.3729 & 1.9352 & -42.6450 & -2.0007 & 27.7195 & 43.7349 & -29.2047 & 5.8138 & -0.3634 & -23.5799 & -3.4108 & 23.7542 \\
\hline 660 & 2.2 & 15 & -16.0398 & 2.0835 & -15.3891 & 1.9333 & -41.6971 & -1.8030 & 26.5155 & 41.3224 & -27.9852 & 5.6071 & -0.3516 & -23.3603 & -3.3780 & 23.5251 \\
\hline 660 & 2.2 & 20 & -16.0705 & 2.0834 & -15.4080 & 1.9306 & -40.6870 & -1.5654 & 25.1437 & 26.5665 & -20.5268 & 4.3560 & -0.2820 & -22.4621 & -3.1765 & 22.3546 \\
\hline 660 & 2.2 & 25 & -16.0916 & 2.0824 & -15.4388 & 1.9309 & -40.3342 & -1.4985 & 24.7107 & 25.6997 & -20.0174 & 4.2582 & -0.2758 & -22.1812 & -3.1323 & 22.0528 \\
\hline 660 & 2.2 & 30 & -16.1225 & 2.0830 & -15.4628 & 1.9297 & -39.9442 & -1.4068 & 24.1787 & 23.4640 & -18.7616 & 4.0265 & -0.2617 & -21.7367 & -3.0403 & 21.5044 \\
\hline 660 & 2.2 & 40 & -16.2270 & 2.0900 & -15.5465 & 1.9312 & -37.8484 & -0.9401 & 21.4283 & 1.2015 & -7.6087 & 2.1721 & -0.1594 & -19.6995 & -2.6238 & 18.9906 \\
\hline 660 & 2.2 & 50 & -16.3486 & 2.0984 & -15.6515 & 1.9338 & -36.9895 & -0.7576 & 20.3284 & -21.4244 & 3.7398 & 0.2826 & -0.0549 & -18.3565 & -2.3839 & 17.4674 \\
\hline 660 & 2.2 & 60 & -16.5134 & 2.1103 & -15.7940 & 1.9373 & -33.2557 & -0.0074 & 15.7181 & -46.7099 & 16.0099 & -1.6927 & 0.0507 & -15.1915 & -1.8062 & 13.8063 \\
\hline 660 & 2.2 & 70 & -16.7301 & 2.1296 & -15.9601 & 1.9397 & -30.5047 & 0.5188 & 12.4297 & -76.0719 & 30.2332 & -3.9798 & 0.1728 & -12.3894 & -1.3418 & 10.7534 \\
\hline 660 & 2.2 & 80 & -17.0743 & 2.1681 & -16.2179 & 1.9457 & -28.2245 & 0.9254 & 9.8460 & -116.4726 & 49.5177 & -7.0368 & 0.3338 & -9.3207 & -0.9038 & 7.6881 \\
\hline 660 & 2.2 & 90 & -17.3836 & 2.2100 & -16.4765 & 1.9450 & -28.7464 & 0.7758 & 10.7484 & -122.6619 & 52.5263 & -7.5212 & 0.3596 & -7.6649 & -0.7847 & 6.5109 \\
\hline 660 & 2.3 & 0 & -15.9946 & 2.0882 & -15.2844 & 1.9275 & -42.1833 & -1.8499 & 26.9852 & 51.2474 & -32.8655 & 6.4029 & -0.3947 & -23.8961 & -3.4245 & 23.9812 \\
\hline 660 & 2.4 & 0 & -16.1798 & 2.1247 & -15.2973 & 1.9305 & -44.2697 & -2.1175 & 29.1167 & 62.1700 & -37.9808 & 7.1711 & -0.4318 & -25.6072 & -3.6861 & 25.8635 \\
\hline 660 & 2.5 & 0 & -16.3614 & 2.1617 & -15.3158 & 1.9358 & -46.7334 & -2.4902 & 31.8049 & 63.2385 & -37.9598 & 7.0546 & -0.4185 & -27.2020 & -3.9418 & 27.6511 \\
\hline 660 & 2.6 & 0 & -16.5160 & 2.1947 & -15.3132 & 1.9385 & -48.2719 & -2.6790 & 33.3545 & 58.8104 & -35.1755 & 6.4784 & -0.3799 & -28.7290 & -4.1957 & 29.3882 \\
\hline 660 & 2.7 & 0 & -16.7545 & 2.2411 & -15.4121 & 1.9580 & -49.8298 & -2.8628 & 34.8902 & 57.1738 & -33.8531 & 6.1607 & -0.3566 & -29.6921 & -4.3461 & 30.4571 \\
\hline 660 & 2.8 & 0 & -16.7545 & 2.2411 & -15.4121 & 1.9580 & -49.8298 & -2.8628 & 34.8902 & 57.1738 & -33.8531 & 6.1607 & -0.3566 & -29.6921 & -4.3461 & 30.4571 \\
\hline 530 & 1.5 & 0 & -15.4483 & 2.0275 & -15.1533 & 1.9529 & -22.3937 & 2.0759 & 2.6127 & -38.6483 & 17.9350 & -2.8595 & 0.1543 & -7.2936 & -0.0297 & 3.0690 \\
\hline
\end{tabular}




\begin{tabular}{|c|c|c|c|c|c|c|c|c|c|c|c|c|c|c|c|c|}
\hline$\lambda$ & $\mathbf{D}_{\mathrm{f}}$ & $f_{\text {organics }}$ & c0 & c1 & g0 & g1 & Ho & H1 & H2 & s0 & s1 & s2 & s3 & k0 & k1 & k2 \\
\hline 530 & 1.5 & 5 & -15.4354 & 2.0230 & -15.1684 & 1.9518 & -29.0070 & 0.4851 & 11.6650 & -12.3844 & 4.3217 & -0.5290 & 0.0223 & -12.7832 & -1.3611 & 10.6311 \\
\hline 530 & 1.5 & 25 & -15.4925 & 2.0170 & -15.2770 & 1.9552 & -21.9488 & 1.9199 & 2.8396 & 10.3025 & -7.6207 & 1.5526 & -0.0978 & -6.2980 & -0.0626 & 2.6354 \\
\hline 530 & 1.5 & 50 & -15.7149 & 2.0309 & -15.4302 & 1.9540 & -19.7990 & 2.4336 & -0.0981 & -35.8653 & 16.1143 & -2.4826 & 0.1292 & -4.8126 & 0.2522 & 0.8143 \\
\hline 530 & 1.5 & 70 & -15.9573 & 2.0492 & -15.6296 & 1.9555 & -22.5647 & 1.7752 & 3.7288 & -40.5993 & 17.1966 & -2.4689 & 0.1189 & -7.0733 & -0.3645 & 4.2926 \\
\hline 530 & 1.6 & 0 & -15.4582 & 2.0301 & -15.1645 & 1.9541 & -30.2522 & 0.2839 & 13.0506 & -13.4329 & 5.3086 & -0.7402 & 0.0353 & -14.1640 & -1.6051 & 12.2251 \\
\hline 530 & 1.6 & 5 & -15.4217 & 2.0195 & -15.2076 & 1.9574 & -23.3446 & 1.5936 & 4.7752 & -49.7193 & 22.9073 & -3.5832 & 0.1883 & -8.0395 & -0.4520 & 4.9281 \\
\hline 530 & 1.6 & 15 & -15.4624 & 2.0151 & -15.2338 & 1.9474 & -24.8580 & 1.2147 & 6.9134 & -18.5029 & 6.1204 & -0.6164 & 0.0157 & -9.4089 & -0.8034 & 6.9306 \\
\hline 530 & 1.6 & 50 & -15.6189 & 2.0175 & -15.4049 & 1.9499 & -20.6727 & 2.0390 & 1.7690 & -44.1478 & 19.1614 & -2.8166 & 0.1389 & -5.3902 & -0.0480 & 2.1902 \\
\hline 530 & 1.6 & 70 & -15.8549 & 2.0337 & -15.6103 & 1.9529 & -23.6874 & 1.4126 & 5.5919 & -41.5504 & 18.4041 & -2.7716 & 0.1403 & -6.9495 & -0.4497 & 4.5236 \\
\hline 530 & 1.7 & 0 & -15.3457 & 2.0117 & -15.1085 & 1.9449 & -22.17 & 1.8602 & 3.2575 & -75.8437 & 36.5469 & -5.9322 & 0.3222 & -6.9526 & -0.1792 & 3.4198 \\
\hline 530 & 1.7 & 1 & -15.4024 & 2.0197 & -15.1596 & 1.9518 & -24.1066 & 1.4635 & 5.6682 & -58.3757 & 27. & -4.3801 & 34 & -8.8238 & -0.5832 & 5.8255 \\
\hline 530 & 1.7 & 5 & -15.3735 & 2.0148 & -15.1536 & 1.9483 & -28.3354 & 0.3895 & 11.6814 & -40.0908 & 17.5 & -2.6200 & 0.1315 & -12.1463 & -1.4425 & 10.6119 \\
\hline 530 & 1.7 & 10 & -15.4378 & 2.0218 & -15.1620 & 1.9477 & -23 & 1.7445 & 4.1842 & -76.1110 & & -6.0263 & 0.3294 & 93 & -0.2535 & 4.0447 \\
\hline 530 & 1.7 & 15 & -15.4566 & 2.0202 & -15.2130 & 1.9520 & -22 & 1.8 & 3.5627 & -71 & 172 & -5.5807 & 31 & -7.2562 & 67 & 3.8218 \\
\hline 530 & 1.7 & 20 & -15.4286 & 2.0140 & -15.2137 & 1.9479 & -29 & 0.1 & 13.1085 & -26 & & -1.3589 & & -13.0825 & 438 & 11.8172 \\
\hline 530 & 1.7 & 25 & -15.5002 & 2.0207 & -15.2542 & 1.9504 & -25.7225 & 1.0879 & 7.8131 & -64.1386 & 29.9268 & -4.7034 & & & 603 & 7.3838 \\
\hline 530 & 1.7 & 30 & -15.5068 & 2.0173 & -15.2676 & 1.9484 & -22.6320 & 1.7391 & 3.8814 & -67.8289 & 32.2766 & -5.1718 & 0.2769 & -7.4854 & -0.3559 & 4.3434 \\
\hline 530 & 1.7 & 40 & -15.6444 & 2.0317 & -15.3452 & 1.9509 & -25.2849 & 1.2893 & 6.8754 & -18.2671 & 7.6862 & -1.1265 & 0.0563 & -9.4102 & -0.6937 & 6.5832 \\
\hline 530 & 1.7 & 50 & -15.6533 & 2.0231 & -15.4243 & 1.9526 & -24.0667 & 1.3764 & 5.8946 & -30.6855 & 13.3950 & -1.9923 & 0.0995 & -8.0697 & -0.5757 & 5.4641 \\
\hline 530 & 1.7 & 60 & -15.7950 & 2.0357 & -15.5214 & 1.9543 & -24.5993 & 1.3187 & 6.3996 & -32.0283 & 14.5091 & -2.2417 & 0.1164 & -8.5442 & -0.6647 & 6.0790 \\
\hline 530 & 1.7 & 70 & -15.8112 & 2.0278 & -15.6123 & 1.9528 & -30.4234 & 0.0299 & 14.0204 & -13.5435 & 5.1912 & -0.6883 & 0.0307 & -12.3873 & -1.5659 & 11.3281 \\
\hline 530 & 1.7 & 80 & -16.0606 & 2.0611 & -15.7484 & 1.9540 & -34.5742 & -0.6042 & 18.5583 & -14.2286 & 5.1097 & -0.6168 & 0.0243 & -14.3195 & -1.8881 & 13.6114 \\
\hline 530 & 1.7 & 90 & -16.0906 & 2.0656 & -15.8622 & 1.9540 & -23.3292 & 1.3332 & 5.9157 & -10.2931 & 3.6915 & -0.4636 & 0.0198 & -5.9208 & -0.5010 & 4.4057 \\
\hline 530 & 1.8 & 0 & -15.3770 & 2.0207 & -15.1030 & 1.9442 & -22.3631 & 1.8124 & 3.5980 & -116.1937 & 55.9503 & -9.0290 & 0.4865 & -6.5949 & -0.1207 & 3.0855 \\
\hline 530 & 1.8 & 5 & -15.4707 & 2.0325 & -15.1466 & 1.9464 & -25.4391 & 1.2396 & 7.2465 & -113.7617 & 54.9201 & -8.8863 & 0.4800 & -9.4615 & -0.6792 & 6.5818 \\
\hline 530 & 1.8 & 50 & -15.6722 & 2.0302 & -15.3889 & 1.9469 & -26.8964 & 0.8227 & 9.4017 & -81.3740 & 38.0949 & -5.9855 & 0.3140 & -10.2878 & -1.0140 & 8.2289 \\
\hline 530 & 1.8 & 70 & -15.8629 & 2.0396 & -15.6026 & 1.9506 & -26.6440 & 0.8021 & 9.3698 & -29.9995 & 12.5153 & -1.7632 & 0.0829 & -9.7750 & -1.0421 & 8.1523 \\
\hline 530 & 1.9 & 0 & -15.3627 & 2.0203 & -15.0710 & 1.9375 & -25.2539 & 1.1174 & 7.6052 & -138.0865 & 65.9737 & -10.5439 & 0.5621 & -9.4218 & -0.7977 & 6.9900 \\
\hline 530 & 1.9 & 5 & -15.3602 & 2.0178 & -15.0659 & 1.9333 & -28.0789 & 0.4681 & 11.3749 & -107.4108 & 50.0976 & -7.8234 & 0.4075 & -12.2964 & -1.4550 & 10.8210 \\
\hline 530 & 1.9 & 25 & -15.4880 & 2.0251 & -15.1747 & 1.9369 & -22.8383 & 1.6702 & 4.3719 & -109.5103 & 51.0049 & -7.9524 & 0.4138 & -7.2266 & -0.3279 & 4.2123 \\
\hline 530 & 1.9 & 50 & -15.6402 & 2.0292 & -15.3707 & 1.9434 & -30.1632 & 0.0449 & 13.9158 & -61.7963 & 27.5115 & -4.1197 & 0.2063 & -12.3549 & -1.5363 & 11.2004 \\
\hline 530 & 1.9 & 70 & -15.9053 & 2.0507 & -15.5866 & 1.9482 & -29.1671 & 0.3470 & 12.3573 & -59.0646 & 26.7554 & -4.0790 & 0.2081 & -10.9436 & -1.2535 & 9.5521 \\
\hline 530 & 2 & 0 & -15.3493 & 2.0228 & -14.9997 & 1.9253 & -28.1476 & 0.4633 & 11.5022 & -111.8723 & 51.4189 & -7.9118 & 0.4065 & -11.9231 & -1.3635 & 10.3555 \\
\hline 530 & 2 & 5 & -15.4374 & 2.0345 & -15.0530 & 1.9303 & -29.2671 & 0.2896 & 12.7102 & -97.4479 & 44.4187 & -6.7869 & 0.3466 & -12.6300 & -1.4759 & 11.1370 \\
\hline 530 & 2 & 25 & -15.5146 & 2.0330 & -15.1524 & 1.9322 & -25.7346 & 1.0450 & 8.1582 & -127.5849 & 59.1440 & -9.1710 & 0.4746 & -9.5137 & -0.8343 & 7.2475 \\
\hline 530 & 2 & 50 & -15.7279 & 2.0467 & -15.3507 & 1.9393 & -26.3697 & 0.9306 & 8.8733 & -132.3193 & 61.4817 & -9.5533 & 0.4953 & -9.2747 & -0.8336 & 7.1611 \\
\hline 530 & 2 & 70 & -15.9225 & 2.0590 & -15.5251 & 1.9379 & -28.8131 & 0.4335 & 11.9367 & -91.6314 & 41.5217 & -6.3004 & 0.3191 & -10.5808 & -1.1770 & 9.1418 \\
\hline 530 & 2.1 & 0 & -15.4277 & 2.0413 & -14.9538 & 1.9170 & -31.3780 & -0.1503 & 15.4513 & -63.5038 & 26.1653 & -3.5666 & 0.1598 & -14.0733 & -1.7711 & 12.9918 \\
\hline 530 & 2.1 & 5 & -15.4687 & 2.0446 & -14.9928 & 1.9200 & -31.5647 & -0.1735 & 15.6231 & -66.1172 & 27.5916 & -3.8238 & 0.1751 & -14.4141 & -1.8410 & 13.4176 \\
\hline 530 & 2.1 & 25 & -15.5506 & 2.0450 & -15.0785 & 1.9202 & -28.9548 & 0.4140 & 12.1588 & -89.2740 & 39.1348 & -5.7337 & 0.2801 & -12.2176 & -1.3728 & 10.6288 \\
\hline
\end{tabular}




\begin{tabular}{|c|c|c|c|c|c|c|c|c|c|c|c|c|c|c|c|c|}
\hline$\lambda$ & $\mathbf{D}_{\mathrm{f}}$ & $f_{\text {organics }}$ & c0 & c1 & g0 & g1 & H0 & H1 & H2 & s0 & s1 & s2 & s3 & k0 & k1 & k2 \\
\hline 530 & 2.1 & 50 & -15.7581 & 2.0580 & -15.2814 & 1.9284 & -27.0473 & 0.8296 & 9.6674 & -125.3823 & 57.0188 & -8.6725 & 0.4403 & -9.8592 & -0.9329 & 7.8765 \\
\hline 530 & 2.1 & 70 & -16.0226 & 2.0821 & -15.4889 & 1.9320 & -28.8712 & 0.4955 & 11.8323 & -117.5095 & 53.1077 & -8.0261 & 0.4048 & -10.4563 & -1.1220 & 8.9375 \\
\hline 530 & 2.2 & 0 & -15.4744 & 2.0543 & -14.8869 & 1.9057 & -32.6405 & -0.3626 & 16.9392 & -24.6864 & 5.7529 & -0.0381 & -0.0411 & -15.3279 & -2.0051 & 14.5306 \\
\hline 530 & 2.2 & 1 & -15.4866 & 2.0559 & -14.8813 & 1.9041 & -34.3386 & -0.7204 & 19.0909 & -23.0105 & 5.1492 & 0.0225 & -0.0423 & -16.4775 & -2.2411 & 15.9679 \\
\hline 530 & 2.2 & 5 & -15.4982 & 2.0558 & -14.9006 & 1.9052 & -34.1257 & -0.6766 & 18.8197 & -25.2534 & 6.2635 & -0.1630 & -0.0319 & -16.1602 & -2.1793 & 15.5842 \\
\hline 530 & 2.2 & 10 & -15.5622 & 2.0638 & -14.9495 & 1.9106 & -33.3220 & -0.4539 & 17.6143 & -31.3818 & 9.4207 & -0.6972 & -0.0022 & -15.2506 & -1.9580 & 14.3336 \\
\hline 530 & 2.2 & 15 & -15.5653 & 2.0607 & -14.9693 & 1.9103 & -31.9414 & -0.1665 & 15.8674 & -33.4950 & 10.3724 & -0.8393 & 0.0049 & -14.7735 & -1.8725 & 13.7811 \\
\hline 530 & 2.2 & 20 & -15.5853 & 2.0599 & -14.9847 & 1.9082 & -31.4042 & -0.0434 & 15.1449 & -52.5260 & 20.0485 & -2.4740 & 0.0967 & -14.1882 & -1.7458 & 13.0338 \\
\hline 530 & 2.2 & 25 & -15.6060 & 2.0596 & -15.0166 & 1.9097 & -30.8199 & 0.0778 & 14.4001 & -53.4761 & 20.5159 & -2.5496 & 0.1007 & -13.7828 & -1.6703 & 12.5548 \\
\hline 530 & 2.2 & 30 & -15.6303 & 2.0601 & -15.0392 & 1.9093 & -30.6155 & 0.1251 & 14.1210 & -52.9759 & 20.3525 & -2.5376 & 0.1009 & -13.6031 & -1.6370 & 12.3484 \\
\hline 530 & 2.2 & 40 & -15.7160 & 2.0667 & -15.1111 & 1.9119 & -29.0655 & 0.4714 & 12.0828 & -72.8733 & 30.2813 & -4.1828 & 0.1914 & -12.0823 & -1.3276 & 10.4756 \\
\hline 530 & 2.2 & 50 & -15.8023 & 2.0727 & -15.1879 & 1.9135 & -29.6 & 0.3 & 12.7851 & -98.7155 & 43.2500 & -6.3430 & 0.3108 & -11.8632 & -1.3104 & 10.3195 \\
\hline 530 & 2.2 & 60 & .9231 & 2.0823 & -15.3025 & 1.9185 & & & 9.9 & -112. & & & & & -0.9560 & 637 \\
\hline 530 & 2.2 & 70 & -16. & 2.0972 & -15.4269 & 1.9226 & -26 & 0.9 & 9.2630 & -11 & 50. & -7. & & 68 & -0.8233 & 7.1374 \\
\hline 530 & 2.2 & 80 & -16.2901 & 2.1253 & -15.5877 & 1.9287 & -26 & 0.9 & 9.4894 & 439 & 47. & 96 & & 556 & -0.7512 & 6.5267 \\
\hline 530 & 2.2 & 90 & -16.3319 & 2.1369 & -15.6593 & 1.9231 & -27 & 0.7 & 10.0927 & -73.9224 & 31.8899 & -4.6050 & & -7.6 & -0.7757 & 6.4075 \\
\hline 530 & 2.3 & 0 & -15.5285 & 2.0646 & -14.8693 & 1.9029 & -32 & -0.2718 & 16.6524 & 277 & 3.4914 & & 78 & -15.4153 & -1.9543 & 14.4179 \\
\hline 530 & 2.4 & 0 & -15.6642 & 2.0932 & -14.8418 & 1.8984 & -34.9362 & -0 & 19.2588 & & 40 & & & & -2.2573 & 319 \\
\hline 530 & 2.5 & 0 & 597 & 2.1177 & -14.7 & 1.8926 & 3215 & & 22.7353 & & -21. & & & 315 & -2.5812 & 157 \\
\hline 530 & 2.6 & 0 & -15.8535 & 2.1392 & -14.7374 & 1.8852 & -40.0848 & -1.5995 & 25.4485 & 49.5473 & -31.6996 & 6.1786 & -0.3816 & -20.5944 & -2.9240 & 20.6830 \\
\hline 530 & 2.7 & 0 & -16.0074 & 2.1717 & -14.7684 & 1.8930 & -42.8377 & -2.1139 & 28.7616 & 69.7092 & -41.8366 & 7.8571 & -0.4735 & -21.9356 & -3.1892 & 22.3479 \\
\hline 530 & 2.8 & 0 & -16.0074 & 2.1717 & -14.7684 & 1.8930 & -42.8377 & -2.1139 & 28.7616 & 69.7092 & -41.8366 & 7.8571 & -0.4735 & -21.9356 & -3.1892 & 22.3479 \\
\hline 467 & 1.5 & 0 & -15.2250 & 2.0243 & -14.9285 & 1.9460 & -21.3605 & 2.1205 & 2.0663 & -35.1209 & 16.2828 & -2.5973 & 0.1403 & -6.4219 & 0.0321 & 2.4416 \\
\hline 467 & 1.5 & 5 & -15.2593 & 2.0374 & -14.9620 & 1.9575 & -20.6890 & 2.5173 & 0.4504 & -2.4744 & -1.8736 & 0.7619 & -0.0666 & -4.6311 & 0.6979 & -0.7219 \\
\hline 467 & 1.5 & 25 & -15.1857 & 2.0120 & -15.0053 & 1.9525 & -23.9461 & 1.2193 & 6.5281 & 25.4720 & -15.2767 & 2.8497 & -0.1714 & -7.9766 & -0.6104 & 5.4794 \\
\hline 467 & 1.5 & 50 & -15.3527 & 2.0269 & -15.0907 & 1.9490 & -19.2520 & 2.3718 & 0.0752 & -41.9971 & 19.6730 & -3.1445 & 0.1693 & -4.2522 & 0.2672 & 0.5329 \\
\hline 467 & 1.5 & 70 & -15.4996 & 2.0464 & -15.1718 & 1.9464 & -16.4574 & 2.8418 & -2.9382 & -58.8100 & 26.4711 & -4.0238 & 0.2051 & -2.5874 & 0.4590 & -0.9002 \\
\hline 467 & 1.6 & 0 & -15.2353 & 2.0268 & -14.9409 & 1.9474 & -28.7077 & 0.4507 & 11.8045 & -3.6309 & 0.3396 & 0.1020 & -0.0122 & -12.9050 & -1.4491 & 11.0611 \\
\hline 467 & 1.6 & 5 & -15.1087 & 2.0092 & -14.9205 & 1.9485 & -23.1181 & 1.4085 & 5.4647 & -74.3275 & 36.0744 & -5.9106 & 0.3245 & -8.1900 & -0.6441 & 5.7107 \\
\hline 467 & 1.6 & 25 & -15.1530 & 2.0094 & -14.9482 & 1.9418 & -27.0336 & 0.4617 & 10.8670 & -7.1065 & 0.0965 & 0.4647 & -0.0496 & -11.0903 & -1.3638 & 9.8096 \\
\hline 467 & 1.6 & 50 & -15.2620 & 2.0145 & -15.0787 & 1.9467 & -23.7455 & 1.1604 & 6.7018 & -38.6478 & 17.0336 & -2.5500 & 0.1282 & -7.8347 & -0.7112 & 5.8602 \\
\hline 467 & 1.6 & 70 & -15.4007 & 2.0318 & -15.1680 & 1.9461 & -25.4695 & 0.8308 & 8.8622 & -17.6725 & 7.4038 & -1.0838 & 0.0541 & -8.3194 & -0.8399 & 6.6743 \\
\hline 467 & 1.7 & 0 & -15.1155 & 2.0073 & -14.8876 & 1.9386 & -22.1811 & 1.6816 & 4.0381 & -31.3774 & 14.5618 & -2.3199 & 0.1250 & -7.1132 & -0.3364 & 4.1005 \\
\hline 467 & 1.7 & 1 & -15.1170 & 2.0132 & -14.8921 & 1.9455 & -26.0870 & 0.7824 & 9.2430 & -47.7187 & 23.0792 & -3.7922 & 0.2096 & -10.6256 & -1.1477 & 8.7725 \\
\hline 467 & 1.7 & 5 & -15.1067 & 2.0121 & -14.8977 & 1.9440 & -30.8519 & -0.4743 & 16.1665 & 7.4308 & -7.4722 & 1.7897 & -0.1275 & -14.4195 & -2.1684 & 14.3606 \\
\hline 467 & 1.7 & 10 & -15.1654 & 2.0187 & -14.8977 & 1.9428 & -19.8279 & 2.3611 & 0.4682 & -87.7277 & 44.3931 & -7.5678 & 0.4318 & -4.8964 & 0.2865 & 0.7859 \\
\hline 467 & 1.7 & 15 & -15.1618 & 2.0143 & -14.9404 & 1.9467 & -23.6818 & 1.3451 & 6.0014 & -53.7600 & 26.1864 & -4.3148 & 0.2382 & -8.2849 & -0.6135 & 5.6836 \\
\hline 467 & 1.7 & 20 & -15.1850 & 2.0187 & -14.9519 & 1.9452 & -31.4590 & -0.5230 & 16.6700 & -16.5877 & 5.7736 & -0.6280 & 0.0187 & -14.6983 & -2.1725 & 14.5528 \\
\hline 467 & 1.7 & 25 & -15.2028 & 2.0171 & -14.9556 & 1.9427 & -25.4709 & 0.9410 & 8.3817 & -22.6606 & 9.0044 & -1.1840 & 0.0500 & -9.5186 & -0.9018 & 7.3789 \\
\hline 467 & 1.7 & 30 & -15.1800 & 2.0103 & -14.9541 & 1.9397 & -23.6609 & 1.3071 & 6.1293 & -84.1306 & 41.7810 & -6.9752 & 0.3890 & -8.4906 & -0.7054 & 6.1423 \\
\hline
\end{tabular}




\begin{tabular}{|c|c|c|c|c|c|c|c|c|c|c|c|c|c|c|c|c|}
\hline$\lambda$ & $\mathbf{D}_{\mathrm{f}}$ & $f_{\text {organics }}$ & c0 & c1 & g0 & g1 & Ho & H1 & H2 & s0 & s1 & s2 & s3 & k0 & k1 & k2 \\
\hline 467 & 1.7 & 40 & -15.3097 & 2.0274 & -15.0127 & 1.9423 & -25.1544 & 1.1019 & 7.6760 & 31.1196 & -17.1699 & 3.0401 & -0.1762 & -9.5844 & -0.8668 & 7.3337 \\
\hline 467 & 1.7 & 50 & -15.2976 & 2.0204 & -15.0740 & 1.9454 & -22.9614 & 1.4066 & 5.4430 & -46.2425 & 21.7884 & -3.4835 & 0.1870 & -7.2677 & -0.5266 & 4.9292 \\
\hline 467 & 1.7 & 60 & -15.3859 & 2.0313 & -15.1169 & 1.9447 & -27.6765 & 0.4446 & 11.3400 & -17.9367 & 7.7740 & -1.1630 & 0.0586 & -10.9685 & -1.3040 & 9.6534 \\
\hline 467 & 1.7 & 70 & -15.3723 & 2.0284 & -15.1650 & 1.9450 & -34.1261 & -1.0020 & 19.8687 & 10.2521 & -6.5287 & 1.2364 & -0.0745 & -15.2400 & -2.3052 & 15.4740 \\
\hline 467 & 1.7 & 80 & -15.5774 & 2.0685 & -15.2111 & 1.9461 & -37.1340 & -1.3591 & 22.8655 & 10.2683 & -6.7329 & 1.2976 & -0.0790 & -15.9493 & -2.3255 & 16.0399 \\
\hline 467 & 1.7 & 90 & -15.3685 & 2.0509 & -15.1912 & 1.9437 & -23.1269 & 1.1428 & 6.8196 & -5.2663 & 1.5274 & -0.1471 & 0.0042 & -6.3543 & -0.6471 & 5.1619 \\
\hline 467 & 1.8 & 0 & -15.7285 & 2.0831 & -15.2932 & 1.9592 & -31.1127 & -0.1752 & 15.4226 & -130.1084 & 61.2659 & -9.6866 & 0.5122 & -11.8049 & -1.4565 & 10.7326 \\
\hline 467 & 1.8 & 5 & -15.1723 & 2.0243 & -14.8433 & 1.9339 & -24.3049 & 1.3340 & 6.4867 & -122.3392 & 61.1171 & -10.2353 & 0.5724 & -8.4259 & -0.5207 & 5.5241 \\
\hline 467 & 1.8 & 25 & -15.2531 & 2.0294 & -14.9630 & 1.9440 & -31.70 & -0.4742 & 16.6739 & -37.6599 & 17.5699 & -2.7950 & 0.1499 & -14.5915 & -2.0714 & 14.1873 \\
\hline 467 & 1.8 & 50 & -15.3357 & 2.0308 & -15.0297 & 1.9378 & -27.1 & 0.5606 & 10.6962 & -58.7503 & 27.6982 & -4.3992 & 0.2337 & -10.5063 & -1.1779 & 8.9745 \\
\hline 467 & 1.8 & 70 & -15.3919 & 2.0345 & -15.1511 & 1.9418 & -28 & 0.1 & 12.9522 & 2.0684 & -3.2605 & 0.8269 & 888 & -11.2729 & -1.4948 & 10.5753 \\
\hline 467 & 1.9 & 0 & -15.1396 & 2.0166 & -14.8460 & 1.9298 & -23.28 & 1.4146 & 5.6731 & -177.0870 & 780 & -14.6465 & 125 & -7.7418 & -0.5058 & 5.1251 \\
\hline 467 & 1.9 & 5 & -15.1009 & 2.0157 & -14.7945 & 1.9253 & -27 & & 11.1 & -101.8639 & & & 89 & -11.3 & -1.3673 & 10.0462 \\
\hline 467 & 1.9 & 25 & -15.1841 & 2.0197 & -14.8990 & 1.9323 & -23 & 1.2 & 6.3 & 0116 & 425 & & & -7.7863 & -0.5803 & 5.4254 \\
\hline 467 & 1.9 & 50 & -15.3412 & 2.0360 & -15.0293 & 1.9368 & & & 15.0 & 5780 & 27 & -2 & & -12.5334 & -1.6966 & 11.9042 \\
\hline 467 & 1.9 & 70 & -15.4933 & 2.0551 & -15 . & 1.9 & & & 14. & 3573 & 08 & & & 05 & -1.5400 & 665 \\
\hline 467 & 2 & 0 & -15.1130 & 2.0169 & -14.7616 & 1.9148 & -25.8479 & 0.7847 & 9.2983 & -129.2946 & 61.6969 & -9.8565 & 0.5259 & -9.7768 & -1.0037 & 7.9982 \\
\hline 467 & 2 & 5 & -15.1751 & 2.0318 & -14.7893 & 1.9236 & -27.7512 & 0.4176 & 11.6102 & -124.5039 & 59.8334 & -9.6403 & 0.5194 & -11.3581 & -1.3220 & 9.9511 \\
\hline 467 & 2 & 25 & -15.2150 & 2.0287 & -14.8585 & 1.9243 & -24.0297 & 1.2301 & 6.7896 & -157.8741 & 76.3599 & -12.3547 & 0.6673 & -7.7562 & -0.5524 & 5.3735 \\
\hline 467 & 2 & 50 & -15.3907 & 2.0468 & -15.0066 & 1.9321 & -26.4881 & 0.6991 & 9.9642 & -161.8839 & 78.3793 & -12.6905 & 0.6858 & -9.1129 & -0.9106 & 7.3901 \\
\hline 467 & 2 & 70 & -15.4694 & 2.0565 & -15.0517 & 1.9249 & -30.4092 & -0.1312 & 15.0224 & -101.0511 & 47.6277 & -7.5203 & 0.3967 & -11.6095 & -1.5002 & 10.8573 \\
\hline 467 & 2.1 & 0 & -15.2008 & 2.0367 & -14.7349 & 1.9094 & -28.8421 & 0.1785 & 13.0826 & -80.4684 & 35.5475 & -5.2334 & 0.2558 & -11.9144 & -1.4465 & 10.7392 \\
\hline 467 & 2.1 & 5 & -15.1910 & 2.0391 & -14.7273 & 1.9126 & -28.8999 & 0.1716 & 13.1464 & -88.4309 & 39.8327 & -5.9982 & 0.3011 & -12.3942 & -1.5521 & 11.3555 \\
\hline 467 & 2.1 & 25 & -15.2680 & 2.0433 & -14.7903 & 1.9126 & -25.5820 & 0.9624 & 8.6347 & -105.0686 & 48.0961 & -7.3573 & 0.3752 & -9.3550 & -0.8579 & 7.3516 \\
\hline 467 & 2.1 & 50 & -15.4216 & 2.0583 & -14.9261 & 1.9188 & -25.2955 & 1.0100 & 8.3313 & -144.4405 & 68.3194 & -10.8100 & 0.5711 & -8.3038 & -0.7025 & 6.2947 \\
\hline 467 & 2.1 & 70 & -15.5940 & 2.0833 & -15.0090 & 1.9172 & -26.8970 & 0.6971 & 10.3489 & -133.0941 & 62.4855 & -9.8108 & 0.5142 & -9.0259 & -0.9162 & 7.5009 \\
\hline 467 & 2.2 & 0 & -15.2165 & 2.0444 & -14.6641 & 1.8972 & -29.7629 & -0.0102 & 14.2948 & -19.1750 & 2.5816 & 0.6214 & -0.0879 & -12.8919 & -1.6601 & 12.0497 \\
\hline 467 & 2.2 & 1 & -15.1990 & 2.0474 & -14.6285 & 1.8974 & -31.8693 & -0.4877 & 17.0880 & -37.4604 & 12.7007 & -1.2323 & 0.0245 & -14.5741 & -2.0293 & 14.2235 \\
\hline 467 & 2.2 & 5 & -15.2217 & 2.0503 & -14.6575 & 1.9010 & -32.1649 & -0.5623 & 17.5039 & -29.3700 & 8.4060 & -0.4772 & -0.0195 & -14.2925 & -1.9765 & 13.8932 \\
\hline 467 & 2.2 & 10 & -15.2632 & 2.0552 & -14.6851 & 1.9035 & -30.8288 & -0.2051 & 15.5548 & -39.6139 & 13.7847 & -1.4087 & 0.0338 & -13.1405 & -1.6815 & 12.2617 \\
\hline 467 & 2.2 & 15 & -15.2330 & 2.0475 & -14.6699 & 1.8981 & -28.7418 & 0.2526 & 12.8496 & -31.1358 & 9.1435 & -0.5693 & -0.0164 & -12.4100 & -1.5347 & 11.3644 \\
\hline 467 & 2.2 & 20 & -15.2836 & 2.0534 & -14.7134 & 1.9019 & -27.9901 & 0.4408 & 11.7992 & -59.4431 & 23.8465 & -3.1090 & 0.1295 & -11.3847 & -1.3029 & 10.0230 \\
\hline 467 & 2.2 & 25 & -15.2842 & 2.0510 & -14.7214 & 1.9004 & -27.7322 & 0.4875 & 11.4924 & -72.1353 & 30.5181 & -4.2737 & 0.1971 & -11.2953 & -1.2954 & 9.9503 \\
\hline 467 & 2.2 & 30 & -15.3153 & 2.0539 & -14.7486 & 1.9018 & -28.1864 & 0.3865 & 12.0852 & -71.8006 & 30.5272 & -4.3065 & 0.2008 & -11.4609 & -1.3426 & 10.2089 \\
\hline 467 & 2.2 & 40 & -15.3567 & 2.0574 & -14.7739 & 1.9002 & -26.3214 & 0.8071 & 9.6421 & -91.1375 & 40.3701 & -5.9726 & 0.2945 & -9.8598 & -1.0011 & 8.1894 \\
\hline 467 & 2.2 & 50 & -15.4536 & 2.0703 & -14.8358 & 1.9036 & -26.8054 & 0.7214 & 10.2167 & -124.3192 & 57.2902 & -8.8383 & 0.4557 & -9.5292 & -0.9475 & 7.8526 \\
\hline 467 & 2.2 & 60 & -15.5219 & 2.0782 & -14.9051 & 1.9076 & -25.7403 & 0.9312 & 8.9352 & -130.0775 & 60.1462 & -9.3065 & 0.4811 & -8.5305 & -0.7861 & 6.7820 \\
\hline 467 & 2.2 & 70 & -15.6158 & 2.0930 & -14.9704 & 1.9112 & -27.6593 & 0.5370 & 11.3666 & -132.7757 & 61.6566 & -9.5787 & 0.4971 & -9.1078 & -0.9501 & 7.6951 \\
\hline 467 & 2.2 & 80 & -15.7060 & 2.1141 & -15.0054 & 1.9116 & -27.5942 & 0.5635 & 11.3242 & -101.5491 & 46.3602 & -7.0800 & 0.3610 & -8.3212 & -0.8496 & 6.9822 \\
\hline 467 & 2.2 & 90 & -15.5041 & 2.1010 & -14.9155 & 1.8988 & -26.4610 & 0.6389 & 10.5841 & -61.9217 & 27.1741 & -3.9960 & 0.1964 & -7.4641 & -0.8129 & 6.4600 \\
\hline
\end{tabular}




\begin{tabular}{|c|c|c|c|c|c|c|c|c|c|c|c|c|c|c|c|c|}
\hline$\lambda$ & $\mathbf{D}_{\mathrm{f}}$ & $f_{\text {organics }}$ & c0 & c1 & g0 & g1 & HO & H1 & H2 & s0 & s1 & s2 & s3 & k0 & k1 & k2 \\
\hline 467 & 2.3 & 0 & -15.2744 & 2.0552 & -14.6573 & 1.8961 & -29.1260 & 0.2391 & 13.1161 & -11.3711 & -1.2798 & 1.2579 & -0.1228 & -12.8534 & -1.5781 & 11.7616 \\
\hline 467 & 2.4 & 0 & -15.3828 & 2.0789 & -14.6223 & 1.8898 & -31.8896 & -0.3003 & 16.5330 & 20.8495 & -18.1860 & 4.1848 & -0.2904 & -14.8163 & -1.9761 & 14.2390 \\
\hline 467 & 2.5 & 0 & -15.4665 & 2.0997 & -14.5745 & 1.8832 & -34.9085 & -0.9250 & 20.3731 & 52.4292 & -34.6302 & 7.0106 & -0.4510 & -16.7000 & -2.3711 & 16.6524 \\
\hline 467 & 2.6 & 0 & -15.5159 & 2.1151 & -14.4968 & 1.8723 & -37.2465 & -1.4072 & 23.3510 & 74.5120 & -46.0470 & 8.9528 & -0.5601 & -18.7793 & -2.8188 & 19.3463 \\
\hline 467 & 2.7 & 0 & -15.6455 & 2.1437 & -14.5196 & 1.8785 & -40.5182 & -2.0967 & 27.5370 & 96.3327 & -57.1947 & 10.8299 & -0.6646 & -20.4577 & -3.1879 & 21.5461 \\
\hline 467 & 2.8 & 0 & -15.6455 & 2.1437 & -14.5196 & 1.8785 & -40.5182 & -2.0967 & 27.5370 & 96.3327 & -57.1947 & 10.8299 & -0.6646 & -20.4577 & -3.1879 & 21.5461 \\
\hline
\end{tabular}

